\title{
UN NUEVO PARADIGMA DE JUSTICIA
}

\section{HERLY LLERENA}

\section{- Gerente general}

Justicia Consciente S.A.C.

\section{RESUMEN}

Todos somos conscientes del repudio de una gran mayoría de peruanos hacia la corrupción institucionalizada en el Sistema de Justicia. También somos conscientes de que sus instituciones, a pesar de la crítica y del clamor por el cambio, no han hecho, lógicamente, mayor cosa por reformarse. Este ensayo propone combatir la corrupción institucionalmente mediante un análisis integral basado en los "niveles de conciencia" de las organizaciones, lo que permitirá, al revisar, por ejemplo, sus características y rasgos, determinar cuáles son las causas o los aspectos vulnerables de las instituciones directamente involucradas, como el Poder Judicial, el Ministerio Público y el Consejo Nacional de la Magistratura. La propuesta incluye introducir en el Sistema de Justicia innovaciones tecnológicas que se utilizan exitosamente en otros sistemas de justicia, como, por ejemplo, el uso del software para resolver conflictos de manera rápida, económica, transparente y segura.

Palabras clave: sistema de justicia, corrupción, niveles de conciencia.

\section{ABSTRACT}

We are all aware of how the majority of Peruvians rejects corruption, entrenched in the national System of Justice. We are also aware of the fact that its institutions, in spite of increasing criticism and a clamor for change, have done practically nothing, logically, in the name of institutional reform. This essay proposes to combat corruption institutionally by analyzing comprehensively the current System of Justice, based on .the "levels of conscience" of organizations, in terms of which, upon revision of their characteristics and features, for example, it would be possible to determine the causes or the vulnerabilities of the institutions directly involved, such as the Judiciary, the Office of the Attorney General, and the National Council of the Magistrate. This proposal includes introducing into the System of Justice technological innovations that have been successfully used in other justice systems, such as a software that helps to resolve conflicts quickly, economically, transparently, and safely.

Keywords: justice system, corruption, levels of conscience.

Al constatar el involucramiento de las figuras más representativas de la política nacional, del empresariado y de la administración de justicia en los actos de corrupción más abyectos, lo dicho por el insigne Manuel Gonzáles Prada, hace exactamente cien años, "el Perú es un cuerpo enfermo, donde se pone el dedo brota la pus" (González Prada, 1894/2007), cobra pleno e indiscutible vigor.

Este diagnóstico lapidario mantiene esa vigencia debido a que la estructura con la que se administra la justicia no ha cambiado sustantivamente desde su creación, hace casi dos siglos.

Es tal la situación, que si un abogado de inicios de la República resucitara, no tendría mayores inconvenientes para ejercer su oficio. Los expedientes de papeles cosidos con los que se establece la verdad legal son los mismos, los códigos sustantivos como el civil y el penal son muy similares y los procesales mantienen las mismas instancias, tipos de recursos, resoluciones, notificaciones, etc.

Lo que no ocurriría si este personaje fuera un médico, ingeniero, profesor, arquitecto o de cualquier otra profesión equivalente. En todos estos campos los cambios han sido tan dramáticos que el desconcierto lo paralizaría inmediatamente. Probablemente intentaría matar moscas con una tablet o celular, al no encontrar su viejo periódico, aunque resulte humorístico.

Es como si el desarrollo se hubiera ocupado de mejorarlo todo, exceptuando la administración de justicia. Son muchas las razones y anécdotas que podrían explicar este patético olvido, pero lo que más importa ahora es explorar qué se puede hacer para salvar la situación y no llegar con el mismo lamento al siguiente siglo.

La historia nos enseña que las grandes transformaciones se hacen a partir de un ideal visionario que cristalice las voluntades y los actos de las colectividades. Martin Luther King en los años sesenta (I have a dream, 2013) condujo a su pueblo con un sueño ¿Cuál es el nuestro?

Necesitamos encender una luz, pues bien, hagámoslo y para ello empecemos por revisar esta valiosa pauta 
de sabiduría que nos alcanza Albert Einstein: “Ningún problema puede ser resuelto en el mismo nivel de consciencia en que se creó" (Frases en inglés, s.f.).

Veamos entonces, en principio, cuál es el significado de nivel de conciencia, para después descubrir el respectivo nivel en que se encuentra actualmente el sistema judicial peruano y, seguidamente, ir a un siguiente nivel para cumplir el cometido de formular una propuesta desde ese siguiente nivel de consciencia.

\section{NIVELES DE CONSCIENCIA}

Uno de los autores más destacados que estudian la conciencia es Robert Kegan, quien dice que esta tiene que ver con la manera como todo individuo percibe su propio "yo", la cual evoluciona con la edad. En el siguiente cuadro se muestra tal evolución de la consciencia a nivel individual:

\section{Tabla 1. Órdenes de Consciencia}

\begin{tabular}{|c|c|c|c|c|c|c|}
\hline & $\begin{array}{c}\text { Orden } 0 \\
\text { Incorporativo } \\
\text { De O a } 2 \text { años }\end{array}$ & $\begin{array}{c}\text { Orden } 1 \\
\text { Impulsivo } \\
\text { De } 2 \text { a } 7 \text { años }\end{array}$ & $\begin{array}{c}\text { Orden } 2 \\
\text { Imperial } \\
\text { De } 7 \text { a } 12 \text { años }\end{array}$ & $\begin{array}{c}\text { Orden } 3 \\
\text { Interpersonal } \\
12 \text { años a más }\end{array}$ & $\begin{array}{c}\text { Orden } 4 \\
\text { Institucional }\end{array}$ & $\begin{array}{c}\text { Orden } 5 \\
\text { Interindividual }\end{array}$ \\
\hline Acontecimiento & $\begin{array}{c}\text { Comienza a } \\
\text { diferenciarse } \\
\text { y aparece una } \\
\text { intencionalidad } \\
\text { propia. }\end{array}$ & $\begin{array}{l}\text { Ha surgido } \\
\text { como una } \\
\text { entidad } \\
\text { separada a } \\
\text { nivel físico y } \\
\text { afectivo. }\end{array}$ & $\begin{array}{l}\text { Comienzan las } \\
\text { operaciones } \\
\text { concretas, } \\
\text { con orden y } \\
\text { categorías } \\
\text { lógicas. }\end{array}$ & $\begin{array}{l}\text { Se hace } \\
\text { parte de una } \\
\text { comunidad } \\
\text { más amplia. }\end{array}$ & $\begin{array}{l}\text { Sale de la fusión } \\
\text { en las relaciones } \\
\text { interpersonales } \\
\text { y adquiere el } \\
\text { poder para } \\
\text { operar sobre } \\
\text { ellas. }\end{array}$ & $\begin{array}{l}\text { Deja atrás el "yo" } \\
\text { como institución } \\
\text { e incluso también } \\
\text { a esta Adquiere } \\
\text { pensamiento } \\
\text { dialéctico. }\end{array}$ \\
\hline Identidad & $\begin{array}{l}\text { "Yo soy } \\
\text { movimientos, } \\
\text { reflejos, } \\
\text { sensaciones." }\end{array}$ & $\begin{array}{l}\text { "Yo soy mis } \\
\text { impulsos. A } \\
\text { nivel social: } \\
\text { soy un niño } \\
\text { y eso es } \\
\text { diferente a ser } \\
\text { adulto." }\end{array}$ & $\begin{array}{l}\text { "Yo soy mis } \\
\text { necesidades, } \\
\text { intereses y } \\
\text { deseos." }\end{array}$ & "Nosotros" & $\begin{array}{l}\text { "Yo soy una } \\
\text { institución que } \\
\text { busca generar } \\
\text { cosas en el } \\
\text { mundo." }\end{array}$ & $\begin{array}{c}\text { "Soy un yo } \\
\text { en constante } \\
\text { transformación". }\end{array}$ \\
\hline
\end{tabular}

Fuente: Kegan (2009)

Ken Wilber, uno de los estudiosos contemporáneos más relevantes de esta materia, reconocido por ello como: "el Einstein de la consciencia", recogió las conclusiones de muchos científicos, historiadores, antropólogos, filósofos, místicos, y psicólogos entre otros, para elaborar un mapa con los diferentes niveles de conciencia propios de los seres humanos. (Wilber, 2007, p. 120).

Este conocimiento de la consciencia individual fue llevado al campo organizacional por Frederick Laloux, quien concluyó que "con cada nuevo estadio de conciencia humana, ha ocurrido también un avance revolucionario en nuestra habilidad de colaborar. Esto ha traído consigo un nuevo modelo de organización. Las organizaciones que hoy conocemos son la expresión de nuestra visión del mundo y de nuestro estadio de desarrollo actuales. Antes hubo otros modelos y la evidencia indica que vendrán más" (Laloux, 2014, p. 26).

De esta manera, puede afirmarse que la consciencia es inherente tanto a los individuos como a las organizaciones y que sus correspondientes niveles son semejantes, tal como puede constatarse en la siguiente matriz. 
Tabla 2. Matriz de Niveles de Consciencia

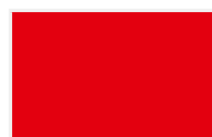

Origen

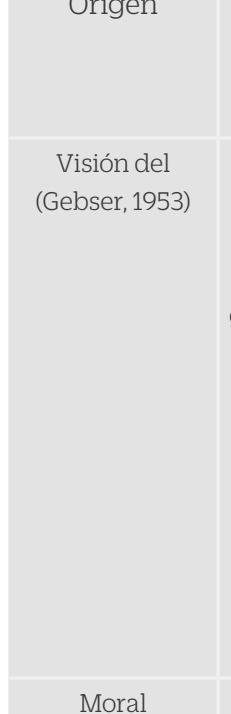

(Kolhberg, 1992)

Estructura cognitiva $^{1}$ (Wilber, 2002)

\section{ROJO (Egocéntrico)}

En algunas tribus aproximadamente 10 mil años a.C.

\section{Mágico - mítico. Centrada en}

"dioses" o "espíritus".

Endiosamiento y glorificación del poder.

Preconvencional.

Es consciente de su individualidad, pero es frágil. Busca seguridad a través del poder, ya sea ejerciéndolo o sometiéndose.

\section{Pre-operacional} conceptual. Aprende y se conecta con otros a través de símbolos, ejemplos y repetición. Tendencia a seguir sólo su propio punto de vista. Inflexibilidad ante algo no aprendido.

\section{ÁMBAR (Etnocéntrico) \\ NARANJA (Mundicéntrico)}

En Mesopotamia hace Con la ilustración a aproximadamente 4 mil años a.C.

mediados del Siglo XVIII.

\section{Mítico. Su identidad, valores colectivos,} estructura jerárquica y sentido de pertenencia, están basados en historias míticas o heroicas.

Convencional. Cree que hay una sola verdad y es la del grupo social al que pertenece.

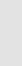

Operacional concreto.

Uso adecuado de la

lógica. Razonamiento

inductivo, de lo general a particular. Resuelven problemas de eventos u objetos concretos.

Falta de desarrollo del pensamiento hipotético, abstracto.

\section{Racional. El}

pensamiento se

hace consciente

de sí mismo, lo

que permite una

identidad individual,

introspectiva,

auto reflexiva

y cosmopolita

que da lugar a

una consciencia

expansiva global.

La razón cosifica y objetiviza el mundo.

Convencional y Postconvencional. Deseo intenso de ser uno mismo, de sobresalir (individualismo).

\section{Operacional} formal. Análisis y primeras formas de Evaluación. El pensamiento no sólo opera en el mundo material concreto, lo hace también sobre el pensamiento mismo. Emerge el razonamiento hipotéticodeductivo.

\section{VERDE (Multi- mundicéntrico)}

Origen: Surgió con el movimiento contracultural de 1960

Pluralista. Considera crucial a la inclusión, en especial a los que están en situaciones desventajosas,

igualdad material y no formal.

Post-convencional. Es "relativista" porque "deconstruye" verdades universales o generalizadas. Sin embargo incluye algunas otras tomadas como principios.

\section{Mente Pluralista o}

Metasistémica. Un individuo puede observar la realidad en términos no sólo lógicos, sino relativos y hasta contradictorios. El conocimiento es un sistema abierto y dinámico, constituido por múltiples dimensiones que interactúan entre sí.

\section{TURQUESA
(Integral)}

Está emergiendo en el presente milenio

Integral. Comprenden profundamente e interactúan, sin juzgarlos, con gente de todos los niveles. A mayor nivel integral, mayor impulso de totalidad. Recurren a grandes datos e imágenes para tener una visión más abarcadora.

Post-convencional tardío. Crean sus propios valores morales de acuerdo con sus convicciones $\mathrm{y}$ vivencias. Tienen principios que tienden a ser comprehensivos, racionales y universalmente aplicables.

Visión-lógica superior o Interparadigmática. Trasciende los sistemas lógicos y busca otros paradigmas distintos de comprensión de la realidad. Estudia los fenómenos que están detrás de cada paradigma, antes de ir tras el "mejor". El conjunto de los paradigmas describen mejor a los fenómenos. 
Tabla 2. Matriz de Niveles de Consciencia (cont.)

\begin{tabular}{|c|c|c|c|c|c|}
\hline & $\begin{array}{c}\text { ROJO } \\
\text { (Egocéntrico) }\end{array}$ & $\begin{array}{c}\text { ÁMBAR } \\
\text { (Etnocéntrico) }\end{array}$ & $\begin{array}{c}\text { NARANJA } \\
\text { (Mundicéntrico) }\end{array}$ & $\begin{array}{c}\text { VERDE } \\
\text { (Multi- } \\
\text { mundicéntrico) }\end{array}$ & $\begin{array}{l}\text { TURQUESA } \\
\text { (Integral) }\end{array}$ \\
\hline $\begin{array}{c}\text { Creencias y } \\
\text { contexto (Beck } \\
\text { \& Cowan, 2005) }\end{array}$ & $\begin{array}{l}\text { La vida es una jungla, } \\
\text { si quieres sobrevivir } \\
\text { tienes que batallar. El } \\
\text { humano es perezoso } \\
\text { por naturaleza, si } \\
\text { quieres que haga algo } \\
\text { tienes que forzarlo o } \\
\text { seducirlo. Para que } \\
\text { la gente haga algo es } \\
\text { preciso usar la fuerza, } \\
\text { el temor o el soborno. }\end{array}$ & $\begin{array}{c}\text { (Azul) La vida es un } \\
\text { regalo para glorificar al } \\
\text { Dador y sus mensajeros. } \\
\text { La alta autoridad diseña } \\
\text { cómo vivir, asigna } \\
\text { posiciones, obligaciones } \\
\text { y dicta normas para que } \\
\text { todos obedezcan. Existe } \\
\text { una forma de más allá y } \\
\text { esta vida es para hacer } \\
\text { los méritos que nos } \\
\text { conduzcan hacia allá. }\end{array}$ & $\begin{array}{c}\text { La vida es un } \\
\text { mercado en el que } \\
\text { los que compiten } \\
\text { exitosamente son } \\
\text { ganadores. La } \\
\text { gente se mueve } \\
\text { por razones } \\
\text { económicas y debes } \\
\text { conocer las reglas } \\
\text { para manipular } \\
\text { en tu beneficio. } \\
\text { La economía es } \\
\text { dirigida por la "mano } \\
\text { invisible”, tiene vida } \\
\text { propia. }\end{array}$ & $\begin{array}{c}\text { La vida es un } \\
\text { organismo en } \\
\text { el que todas sus } \\
\text { partes deben estar } \\
\text { saludables. Se trabaja } \\
\text { para tener contacto } \\
\text { humano, aprender } \\
\text { y conocerse a sí } \\
\text { mismo. Pertenecer, } \\
\text { ser gustado, incluido } \\
\text { y aceptado es más } \\
\text { importante que los } \\
\text { premios económicos. }\end{array}$ & $\begin{array}{c}\text { La vida es un arte, un } \\
\text { desempeño creativo } \\
\text { que emerge por } \\
\text { todos lados. El trabajo } \\
\text { significativo sirve a } \\
\text { toda la vida. El universo } \\
\text { es una sola entidad de } \\
\text { fuerzas elegantemente } \\
\text { equilibradas y } \\
\text { entrelazadas. Nadie es } \\
\text { dueño de la verdad. Con } \\
\text { el conjunto de verdades } \\
\text { se esboza una verdad } \\
\text { más abarcadora. }\end{array}$ \\
\hline $\begin{array}{c}\text { Cognición } \\
\text { social (Selman, } \\
\text { 2003) }\end{array}$ & $\begin{array}{c}\text { O. Perspectiva } \\
\text { Indiferenciada. } \\
\text { Carecen de la facultad } \\
\text { de distinguir su } \\
\text { propia interpretación } \\
\text { y la de otros frente } \\
\text { a una situación } \\
\text { social determinada. } \\
\text { Tampoco pueden } \\
\text { comprender que su } \\
\text { concepción pudiera no } \\
\text { ser correcta. }\end{array}$ & $\begin{array}{l}\text { 1. Perspectiva } \\
\text { diferenciada o subjetiva. } \\
\text { Alcanzan a comprender } \\
\text { que otros pueden } \\
\text { tener perspectivas } \\
\text { diferentes, pero aún no } \\
\text { comprenden las razones } \\
\text { de otros puntos de vista. }\end{array}$ & $\begin{array}{l}\text { 2. Perspectiva } \\
\text { autoreflexiva. } \\
\text { Son capaces de } \\
\text { diferenciar otras } \\
\text { perspectivas } \\
\text { distintas y pueden } \\
\text { reflexionar sobre } \\
\text { su propia conducta, } \\
\text { motivados por esas } \\
\text { otras perspectivas. }\end{array}$ & $\begin{array}{l}\text { 3. Perspectiva mutua } \\
\text { o de tercera persona. } \\
\text { Puede ver su propia } \\
\text { perspectiva, la de } \\
\text { los otros y a la vez } \\
\text { ser alguien neutro } \\
\text { como observador en } \\
\text { tercera persona. }\end{array}$ & $\begin{array}{c}\text { 4. Perspectiva a } \\
\text { profundidad. Es } \\
\text { consciente de que los } \\
\text { motivos, acciones, } \\
\text { pensamientos y } \\
\text { sentimientos de los } \\
\text { otros obedecen a } \\
\text { factores psicológicos. } \\
\text { Se dan cuenta de que } \\
\text { la personalidad es un } \\
\text { sistema de rasgos, } \\
\text { creencias, valores y } \\
\text { actitudes con su propia } \\
\text { historia evolutiva. }\end{array}$ \\
\hline $\begin{array}{l}\text { Organización } \\
\text { (Beck \& Cowan, } \\
\text { 2005) }\end{array}$ & $\begin{array}{l}\text { Quienes demuestran } \\
\text { superioridad tienen el } \\
\text { "poder" de organizar } \\
\text { a los demás que se } \\
\text { consideran inferiores } \\
\text { y se hace lo que } \\
\text { dispone el Gran Jefe. } \\
\text { Esto produce las } \\
\text { desigualdades Tener/ } \\
\text { no tener, poder/no } \\
\text { poder. }\end{array}$ & $\begin{array}{l}\text { (Azul) Jerárquicas } \\
\text { y estructuradas } \\
\text { rígidamente. El poder } \\
\text { está en la posición no } \\
\text { en la personalidad. } \\
\text { Desarrollan } \\
\text { estrategias para crear } \\
\text { sistemas estables y } \\
\text { proteccionistas de sus } \\
\text { miembros. Se valora la } \\
\text { lealtad. }\end{array}$ & $\begin{array}{c}\text { Administración } \\
\text { pragmática con } \\
\text { procedimientos } \\
\text { normados y } \\
\text { medibles. El sistema } \\
\text { funciona a través } \\
\text { de jerarquías de } \\
\text { mérito (meritocracia) } \\
\text { con incentivos } \\
\text { económicos y de } \\
\text { posición por logros. }\end{array}$ & $\begin{array}{l}\text { Priman los equipos } \\
\text { y las relaciones } \\
\text { horizontales, no } \\
\text { La comunicación } \\
\text { abierta y frecuente } \\
\text { reemplaza las } \\
\text { jerarquías. } \\
\text { Promueven los } \\
\text { derechos humanos, } \\
\text { la inclusión, la } \\
\text { diversidad y el } \\
\text { igualitarismo. }\end{array}$ & $\begin{array}{c}\text { Funciones holográficas } \\
\text { e integradas. Todos } \\
\text { son responsables de } \\
\text { todos los objetivos, y } \\
\text { desde discretos pedazos } \\
\text { fractales se defiende al } \\
\text { todo con autonomía. } \\
\text { La información } \\
\text { fluye a través de un } \\
\text { mínimo de filtros o } \\
\text { límites funcionales o } \\
\text { territoriales. }\end{array}$ \\
\hline $\begin{array}{l}\text { Ejemplos } \\
\text { actuales }\end{array}$ & $\begin{array}{c}\text { La mafia, pandillas } \\
\text { callejeras, milicias } \\
\text { tribales. }\end{array}$ & $\begin{array}{l}\text { Iglesias, fuerzas } \\
\text { armadas, mayoría } \\
\text { de instituciones de } \\
\text { gobierno, sistemas de } \\
\text { educación pública. }\end{array}$ & $\begin{array}{l}\text { Empresas } \\
\text { multinacionales, } \\
\text { escuelas de } \\
\text { negocios, etc. }\end{array}$ & $\begin{array}{c}\text { Empresas como } \\
\text { Southwest Airlines, } \\
\text { Ben \& Jerry's, } \\
\text { diversas ONG's. }\end{array}$ & $\begin{array}{c}\text { Organizaciones Teal } \\
\text { (FAVI, Buurtzorg, Sun } \\
\text { Hidraulics, Morning Star, } \\
\text { etc.) }\end{array}$ \\
\hline
\end{tabular}




\section{UTILIDAD DE LA MATRIZ DE CONSCIENCIA}

Este "mapa" permite conocer los diferentes niveles de conciencia en los que puede encontrarse una persona u organización, así como los estadios superiores o más profundos a los que puede accederse.

En esa línea, así como resulta insano que alguien de 40 ó 50 años de edad posea una estructura cognitiva o moral correspondiente a un niño de 10 años, también lo es que una organización se quede estancada permanentemente en niveles básicos.

Bajo estas premisas, es posible por ejemplo, indagar el nivel de consciencia en que se encuentra el poder judicial peruano. Para ello solo hace falta verificar la piedra angular de su creación que es el artículo 158 de la Constitución Política, del cual se desprende que se trata de una institución absolutista, por su pretensión de autonomía suprema y que tiene además una organización vertical y con cadena de mando. Con estas constataciones sabemos que se trata inequívocamente de una organización ámbar.

Esto explica el porqué creen, míticamente, que existe una sola verdad y que es la que ellos poseen (la verdad legal). También explica el porqué reducen los conflictos y la realidad compleja a decisiones basadas en interpretaciones literales de la norma, al utilizar su pensamiento operacional concreto. A ello se suman los exagerados formulismos en sus procedimientos y las parafernalias para distinguir jerarquías, tales como atuendos, medallas y palacios medioevales.

Esta realidad está más allá de cualquier juicio que se pueda hacer sobre el particular puesto que un ámbar sólo puede ofrecer aquello que tiene, como un niño de 10 años o un adolescente a los que no se les puede pedir virtudes de un adulto. De allí que la institución no cambiará a menos que vaya a un siguiente nivel de consciencia.

Por otro lado, también es pertinente advertir que un inconveniente mayúsculo que ofrecen todos los niveles de conciencia, excepto el integral o turquesa, es que cada uno de ellos considera ser poseedor de la verdad absoluta, por lo que son tremendamente displicentes con los demás niveles. Esta es la causa por la que les resulta sumamente complicado evolucionar a un siguiente nivel.

Este es el motivo más relevante por el que el sistema judicial peruano se afinca en la permanencia indefinida de este nivel ámbar y rechaza cualquier intento de cambio o evolución. Por eso es que los intentos de sendas reformas desde hace casi dos siglos han alcanzado sólo lo epidérmico.

\section{UNA MIRADA INTEGRAL}

Frente a todo este panorama, últimamente, se está erigiendo una mirada desde un nivel de consciencia más profundo que es el turquesa - integral, cuya característica más notable es que incluye y trasciende a los anteriores. Es decir, reconoce el valor y la vigencia de los otros niveles como etapas necesarias dentro de un recorrido; pero reconoce también, a la vez, la existencia de un nivel más abarcante y completo, similar a la madurez que incluye y trasciende a la niñez y la adolescencia.

Sin embargo, no se puede continuar con el abordaje del punto sino precisamos de qué hablamos cuando hablamos de integral.

Para ello recurriremos a la Teoría Integral de Ken Wilber que ofrece un mapa para explicar lo integral denominado OCON, acrónimo de OmniCuadrante y OmniNivel². (Wilber, 2005, p. 110) y se representa en la siguiente figura:

\section{Figura 1. Mapa de la Teoría Integral}

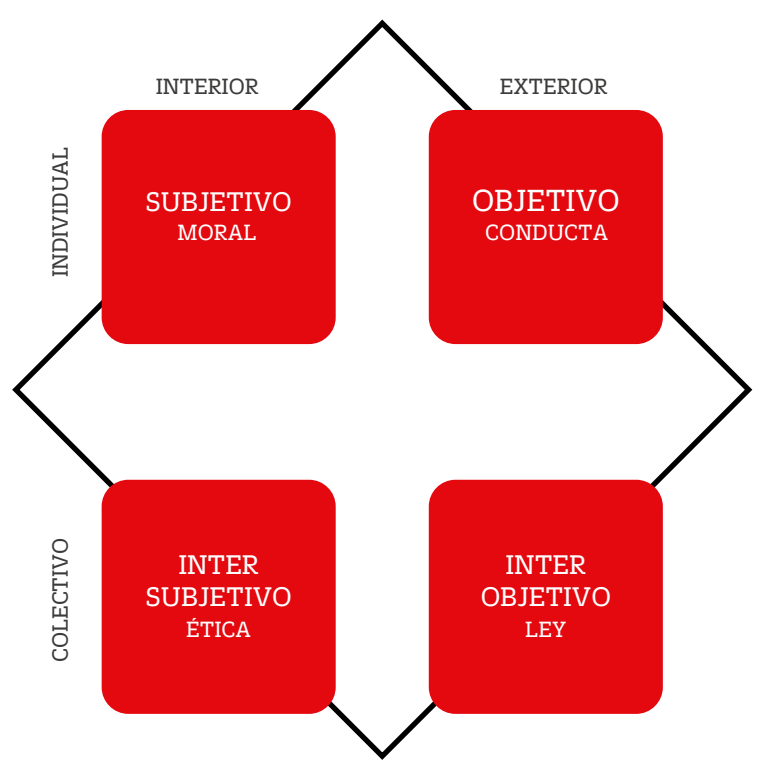

De acuerdo con este mapa, todos los individuos y entidades poseen un aspecto "subjetivo", en el que subyacen los pensamientos, sentimientos, valores morales, visión del mundo, identidad psicosexual, etc. Asimismo, tienen un aspecto "objetivo", constituido por el cuerpo físico, células, tejidos, bioquímica, etc., así como por sus manifestaciones, como es la conducta. Por otro lado, también poseen una parte en la que se encuentra lo "intersubjetivo", es decir la cultura, la ética, los valores familiares, los influjos sociales, la historia, los idiomas, etc. Finalmente, se posee un aspecto "interobjetivo" en el que se encuentran relaciones y sistemas, como por ejemplo, el hogar, el barrio, la ciudad, el país, la familia, centro de trabajo, etc. 


\section{PROPUESTA DE UN NUEVO PARADIGMA DE JUSTICIA:}

Valiéndonos de este enfoque que nos permite tener una noción verdaderamente integral del sistema de justicia, vamos a pasar a avizorar un nuevo paradigma del sistema judicial en el país, desde cada uno de sus respectivos cuadrantes:

\section{CUADRANTE SUBJETIVO:}

Habida cuenta de que la capacidad de afrontar retos y dificultades es directamente proporcional al nivel de consciencia, la transformación del nuevo sistema de justicia comenzará por el cambio de los operadores de dicha transformación.

Si los retos y exigencias que se tienen a la vista son de la más alta envergadura, lo correspondiente es que quienes se encarguen de afrontar ello sean los individuos que tengan las capacidades y potencialidades más refinadas.

En tal sentido, los directivos, jueces y fiscales y demás integrantes de todos los organismos del sistema de justicia, adoptarán y desarrollarán las competencias correspondientes a los niveles de consciencia más profundos, al menos naranja o verde; además de mantener y desarrollar sus conocimientos profesionales.

Además, todos los integrantes del sistema judicial y, particularmente sus líderes, adoptarán el desarrollo de consciencia como un estilo de vida a fin de replicar su evolución en su labor cotidiana.

De esta manera, entre otras bondades, todos ellos serán observantes de una integridad al poseer una coherencia entre sus valores morales y éticos, su conducta y la observancia de la Ley (ver Figura 1); y se evitarán cosas como la que está ocurriendo ahora: nada menos que con el marco conceptual y legal medular de la lucha anticorrupción del Gobierno, en cuya norma que establece medidas para fortalecer la integridad pública y lucha contra la corrupción ${ }^{3}$, se define el término "integridad" como: "Uso adecuado de fondos, recursos, activos y atribuciones en el sector público, para los objetivos oficiales para los que se destinaron".

Como podrá advertirse, han reducido escandalosamente el significado de la palabra integridad a un mero aspecto económico relacionado con el peculado y con un vulgar tráfico de influencias, omitiendo clamorosamente alguna mención a los atributos que constituyen la integridad integral. Eso explica por qué los corruptos admiten con el mayor desparpajo haber cometido sus tropelías mencionando al final la consabida frase: "reconozco estar en falta, pero no cometí ningún delito" o "mentí, pero eso no es ilegal".

\section{CUADRANTE OBJETIVO:}

La estructura física tradicional de la administración de justicia con sus palacios neoclásicos y oficinas ófricas atiborradas de expedientes, junto a inaccesibles jueces y apergaminados abogados, será reemplazada por una moderna, ágil y asequible estructura "tecnolegal".
La tecnología está dando asombrosas soluciones en todas los campos del quehacer humano y la justicia no es la excepción. He aquí algunas luces. Modria es una empresa pionera en la resolución de conflictos online que, desde 2011, resolvió satisfactoriamente más de 400 millones de disputas de clientes insatisfechos de las empresas eBay y PayPal (Oppenheimer, 2018, p. 166). En un 90\% de estos casos no hubo ninguna intervención humana.

El número de casos resueltos por Modria es tres veces mayor que el total de juicios que se ventilan en todas las cortes de Estados Unidos. Entonces ¿cuál sería el inconveniente para que también pueda resolver procesos judiciales comunes en todo el mundo?

A finales del 2017 Modria fue comprada por la empresa Tyler Technologies (Tyler Technologies, s.f.) y se están disponiendo a brindar servicios asequibles a millones de litigantes de diferentes cortes y tribunales de justicia.

Junto a casos como el citado, existen otros como Kleros (Ast \& Lesaege, 2017) que tiene propuestas muy ingeniosas para resolver disputas de manera rápida, económica, transparente y segura, utilizando la plataforma Blockchain, que es considerada como la siguiente ola disruptiva después del Internet.

Estos desarrollos tecnológicos no son aislados; vienen con el vertiginoso e incontrolable avance del dataísmo o religión de los datos (Harari, 2015, p. 400) que está cambiando dramáticamente el futuro de la humanidad a partir del flujo de algoritmos cada vez más sofisticados. Desde que en la biología se llegó a la conclusión de que los organismos, las emociones y los pensamientos no son otra cosa que algoritmos (Harari, 2015, pp. 377-414), la informática ha hecho posible que todas las disciplinas científicas se comuniquen en este mismo idioma de datos y borren sus fronteras, con lo cual están incrementando vertiginosamente sus posibilidades de desarrollo.

No estamos ante algo que quizá ocurrirá en el futuro; son soluciones que están en el presente. Por tanto, la pregunta no es si se impartirá o no justicia a través de plataformas digitales. La pregunta es ¿Qué se espera para adoptarla inmediatamente?

Se calcula que en 5 años el 70 \% de los trabajos serán impactados sustancialmente por la tecnología (Oppenheimer, 2018, p. 11), sea que hagamos algo o no. ¿No sería conveniente migrar cuanto antes y de lleno hacia la automatización tecnológica a fin de ocuparse también a tiempo de las correspondientes reconversiones laborales?

\section{CUADRANTE INTERSUBJETIVO:}

Es imperativo que un sistema de justicia redefina su propósito esencial. Aquel designio tradicional de ser un "poder" estatal independiente y autónomo (Poder Judicial del Perú, s.f.), requiere ser actualizado por otro que incluya la responsabilidad primordial de impulsar el desarrollo evolutivo de conciencia de todos los involucrados.

Cada acto, cada sentencia y la jurisprudencia que este nuevo sistema genere, tendría que tener en cuenta dicha responsabilidad primordial y construir, con base en ello, 
una nueva sociedad más armónica, solidaria y pacífica.

La justicia actual se circunscribe a atribuir la razón legal a una de las partes en conflicto. Esto ocurre tanto en el campo penal, con el fiscal que pugna por una mayor sanción y el delincuente que busca evadir su responsabilidad, como en las otras especialidades (civil, constitucional, comercial, administrativo, laboral, etc.) en las que las partes entran en contienda para hacer prevalecer sus respectivos argumentos.

El efecto de esta dinámica es sumamente perniciosa, desde la perspectiva de la evolución, ya que fomentan furibundos combates egocéntricos y mantienen perpetuamente a todos los involucrados en un nivel de conciencia rojo ámbar, con lo cual el más avezado criminal no se diferencia mucho del más encumbrado juez.

Solamente si llega a insertarse en la cultura jurídica este propósito esencial distinto, podrá romperse aquel círculo vicioso y erigirse, en la práctica, la sociedad justa, armoniosa y pacífica que todos soñamos, porque será el reflejo de aquellos seres humanos con niveles de conciencia profundos como el turquesa - integral, cuyo hábitat natural es éste.

\section{CUADRANTE INTEROBJETIVO:}

En correspondencia con lo expresado en lo intersubjetivo, al nuevo sistema de justicia le corresponderá reformular completamente su estructura. Se prevé que tendrán que existir al menos las siguientes áreas:

- Legislación: solamente las normas de creación constitucional y su respectiva Ley Orgánica estarían en manos del Congreso de la República o del Congreso Constituyente, si fuera el caso. La expedición de las demás normas de desarrollo estaría bajo la competencia exclusiva de un nuevo despacho de mayor nivel del sistema de justicia.

- Regulación: Este mismo despacho estará encargado de cumplir y hacer cumplir lo establecido por ley, así como los objetivos de cobertura de servicios de justicia en todo el país, de acuerdo con estándares, metas e indicadores de gestión previamente establecidos.

- Ejecución: los roles de atención de procesos judiciales, penitenciario y de desarrollo de consciencia podrían ser atendidos, en principio, por el sector privado y, de ser el caso, por sistemas mixtos, a considerar.

- Control: este rol sería competencia exclusiva del Estado a través de sus respectivos mecanismos.

En todas estas áreas, las estructuras, prácticas y procesos del sistema de justicia futuro, tendrán inspiración en las experiencias exitosas que las organizaciones pioneras turquesa - integral nos vienen alcanzando (Laloux, 2014, p. 232) y que autores como Brian Robertson (Robertson, 2015, p. 47) los están traduciendo en métodos, como la holacracia, cuya adopción por las empresas más renombradas del mundo es cada vez más frecuente.

\section{CONCLUSIÓN}

Hoy se sabe, por la ciencia, que la justicia ha sido y seguirá siendo la mejor herramienta para resolver nuestros dilemas sociales y que se ha formado a lo largo de millones de años de evolución (Harari, 2018). Por tanto, no es una definición, un tótem, ni mucho menos un vetusto aparato burocrático anquilosado e inmutable.

Dichos dilemas sociales empezaron en la sabana africana y por siglos se afincaron dentro de las fronteras de naciones y países, hasta este momento en que tenemos en frente problemas universales como el calentamiento global, desigualdad, migraciones, amenazas de guerra nuclear, etc. Está claro que en este nuevo contexto, la justicia que sirvió para dirimir la propiedad de unos metros de tierra o la cría de un ganado en disputa, servirá muy poco.

Por tales razones, se precisa con urgencia actualizar la justicia en fondo y en forma, para lo cual será necesario utilizar un sistema operativo actualizado que permita manejar las complejidades que el nuevo entorno plantea, como la teoría integral que ha sido expuesta. Estas son algunas pinceladas gruesas que ojalá alcancen a delinear, al menos, el perfil de un nuevo paradigma para el sector justicia.

Habrán algunos que acepten la propuesta y otros que lo juzguen de etéreo o fantasioso. A los primeros les recuerdo que iquien sabe a dónde ir, se encuentra a la mitad del camino! A los segundos, lo que decía San Agustín: "para ir donde no se sabe, hay que ir por donde no se sabe".

A todos los insto a sumar y fundirnos en la forja de un nuevo designio visionario para la justicia del Siglo XXI.

\section{REFERENCIAS}

Ast, F., \& Lesaege, C. (2017, 20 de setiembre). Kleros, un protocolo de justicia para Internet. Medium. Obtenido en https://medium.com/kleros/kleros-un-protocolo-dejusticia-para-internet-920c28a588f1

Beck, D. E., \& Cowan, C. C. (2005). Spiral dynamics: Mastering values, leadership and change. New Jersey, NJ: Wiley Blackwell.

Frases en inglés. (s.f.). Obtenido en http://www.frases-ingles. net/aforismos/albert-einstein/

Gebser, J. (1953). Origen y Presente. Girona, España: Ediciones Atalanta.

González Prada, M. (2007). Pájinas Libres. Barcelona, España: Linkgua S. L. (Obra original publicada en 1894).

Harari, D. N. (2015). Homo Deus. London, England: Harvill Secker.

Harari, Y. N. (2018). 21 lecciones para el siglo XXI. New York, NY: Penguin Random House.

I have a dream. (2013, 28 de agosto). La Vanguardia. Obtenido en https://www.lavanguardia.com/ hemeroteca/20130828/54379767638/derechoscivilespolitica-minorias-afroamericanos-negrosmarcha-sobrewashington-discursos.htm 
Kegan, R. (2009). The Evolving Self. Cambridge, MA: Harvard University Press.

Kolhberg, L. (1992). Psicología del Desarrollo Moral. Bilbao, España: Desclée de Brower S.A.

Laloux, F. (2014). Reinventando las organizaciones. Cómo crear organizaciones inspiradas en el siguiente estadio de consciencia humana. Bruselas, Bélgica: Nelson Parker.

Oppenheimer, A. (2018). jSálvese Quien Pueda!. Bogotá, Colombia: Nomos.

Poder Judicial del Perú. (s.f.). Definiciones. Obtenido en https://historico.pj.gob.pe/conocenos. asp?tema=definiciones

Robertson, B. (2015). Holacracia. Un nuevo sistema organizativo para un mundo en continuo cambio. Barcelona, España: Urano.

Selman, R. (2003). The Promotion of Social Awareness. Nueva York, NY: Russell Sage Foundation.

Tyler Technologies. (s.f.). Solutions for courts \& justice. Obtenido en https://www.tylertech.com/solutions/ courts-public-safety/courts-justice

Wilber, K. (2002). Una Visión Integral de la Psicología. Ciudad de México, México: Alamah.

Wilber, K. (2005). Breve Historia de Todas las Cosas (5ta ed.). Barcelona, España: Kayrós.

Wilber, K. (2007). Espiritualidad Integral. Barcelona, España: Kayrós. 